\title{
Amphiphilic star polypept(o)ides as nanomeric vectors in mucosal drug delivery
}

Dimitrios Skoulas,,$^{\sharp, \S}$ Vivien Stuettgen, ${ }^{\#, \S}$ Rachel Gaul, ${ }^{\$}$ Sally-Ann Cryan ${ }^{\$, \S, \dagger}$ David J. Brayden,,$^{\#,, *}$ Andreas Heise $e^{\dagger, \xi, t, *}$

* Department of Chemistry, Royal College of Surgeons in Ireland, 123 St. Stephens Green, Dublin 2, Ireland. "School of Veterinary Medicine and Conway Institute, University College Dublin, Veterinary Science Centre, Belfield, Dublin 4, Ireland. ${ }^{\$}$ School of Pharmacy and Biomolecular Sciences and Tissue Engineering Research Group, Royal College of Surgeons in Ireland, 123 St. Stephen's Green, Dublin 2, Ireland §Science Foundation Ireland (SFI) Centre for Research in Medical Devices (CURAM). 'AMBER, The SFI Advanced Materials and Bioengineering Research Centre.

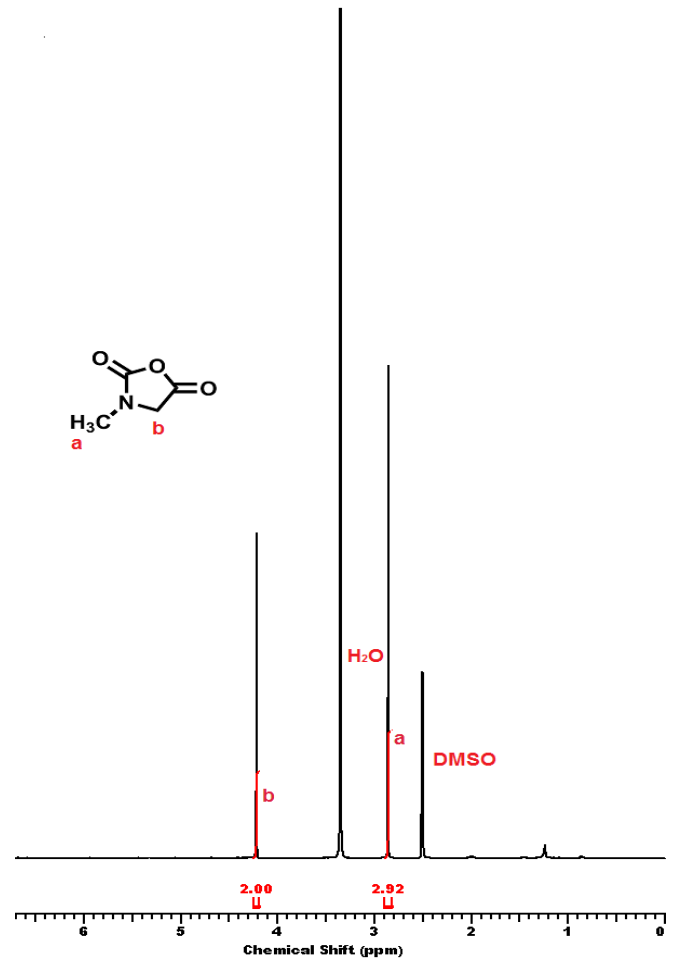

Figure S1. ${ }^{1} \mathrm{H}-\mathrm{NMR}$ spectrum of Sar NCA. 


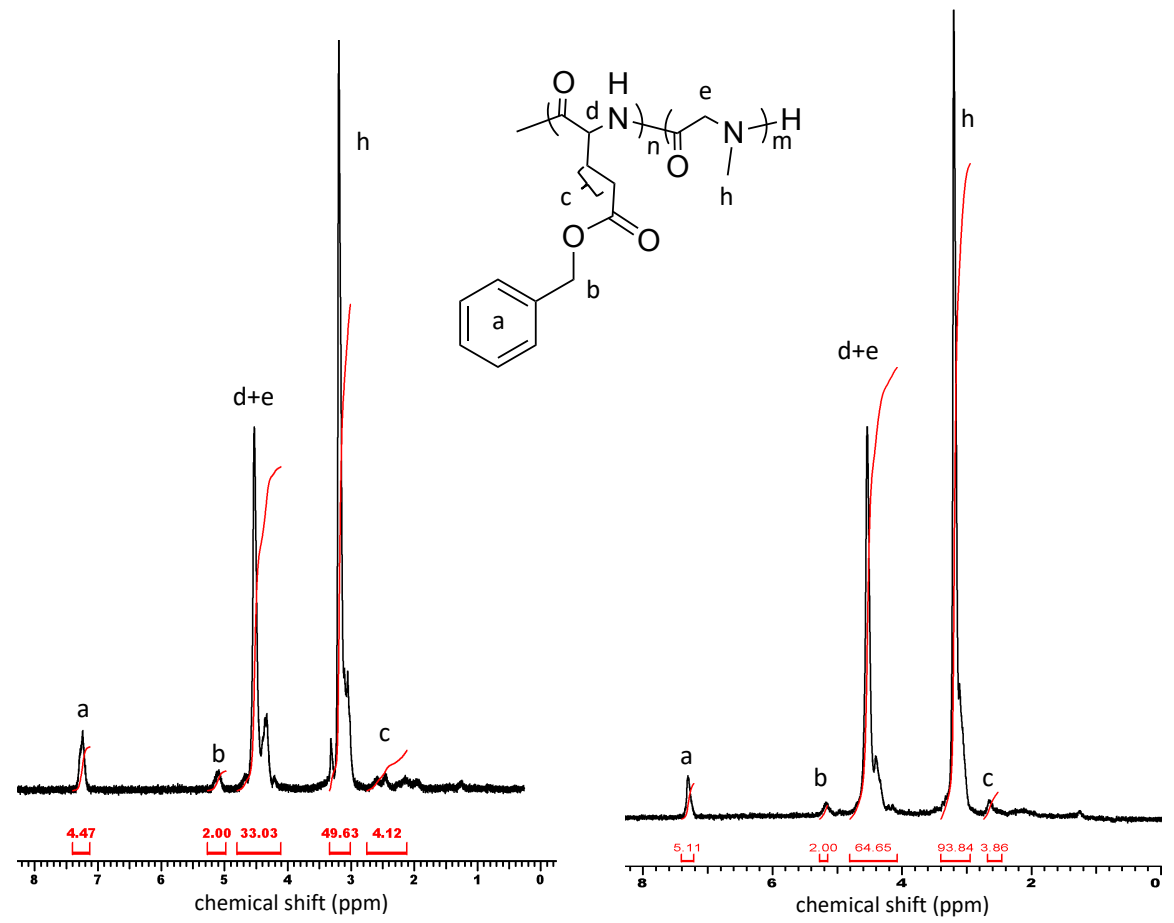

Figure S2. Representative ${ }^{1} \mathrm{H}-\mathrm{NMR}$ spectra of S10 (PBLG $\left.20-b-\mathrm{PSar}_{320}\right)$, left, and S11 (PBLG $20-b$ PSar 640 ), right, in TFA.
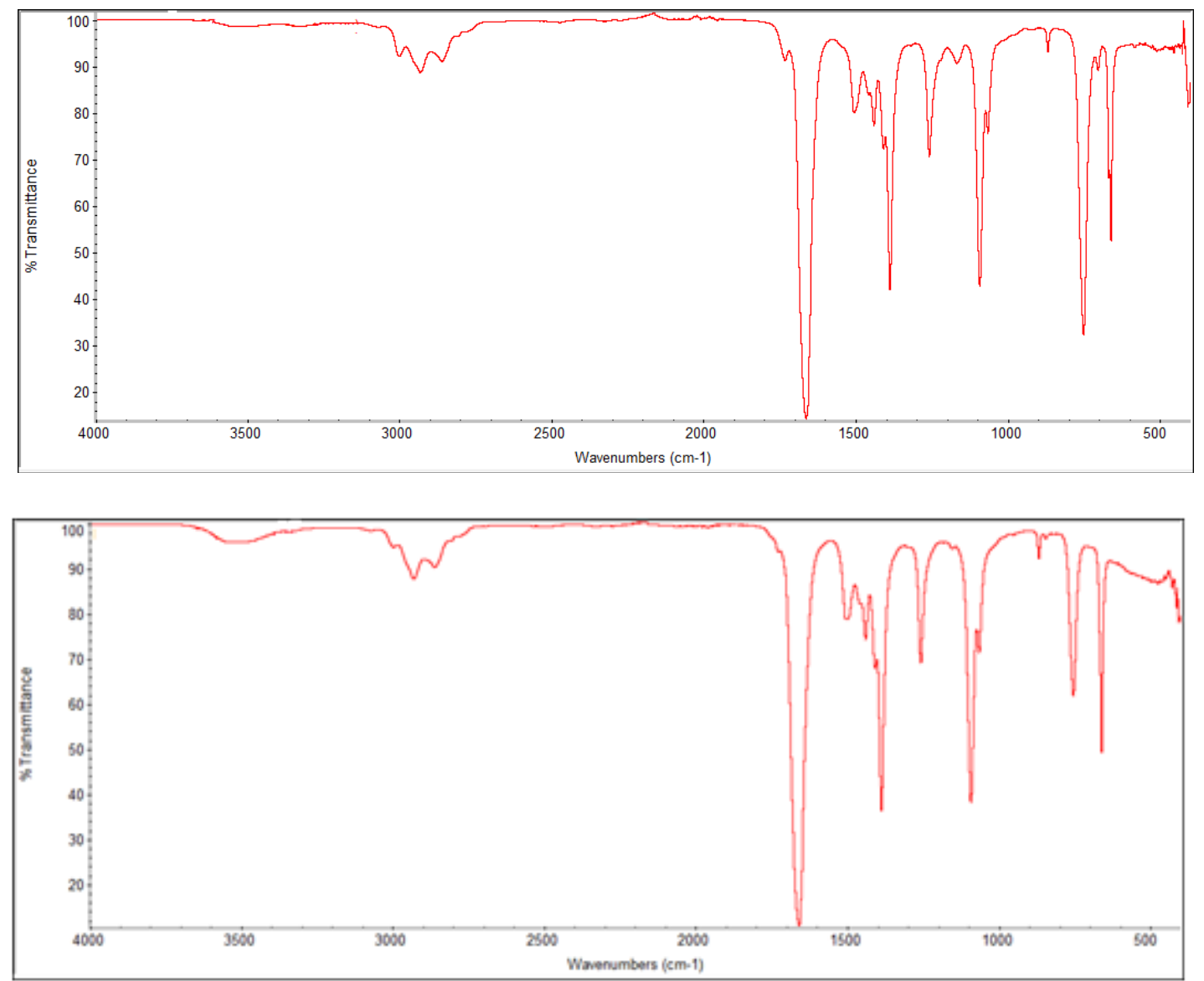

Figure S3. FTIR spectra of 8-arm starPBLG 20 (top) and starPBLG ${ }_{20}-b-$ PSar $_{640}$ (bottom). 

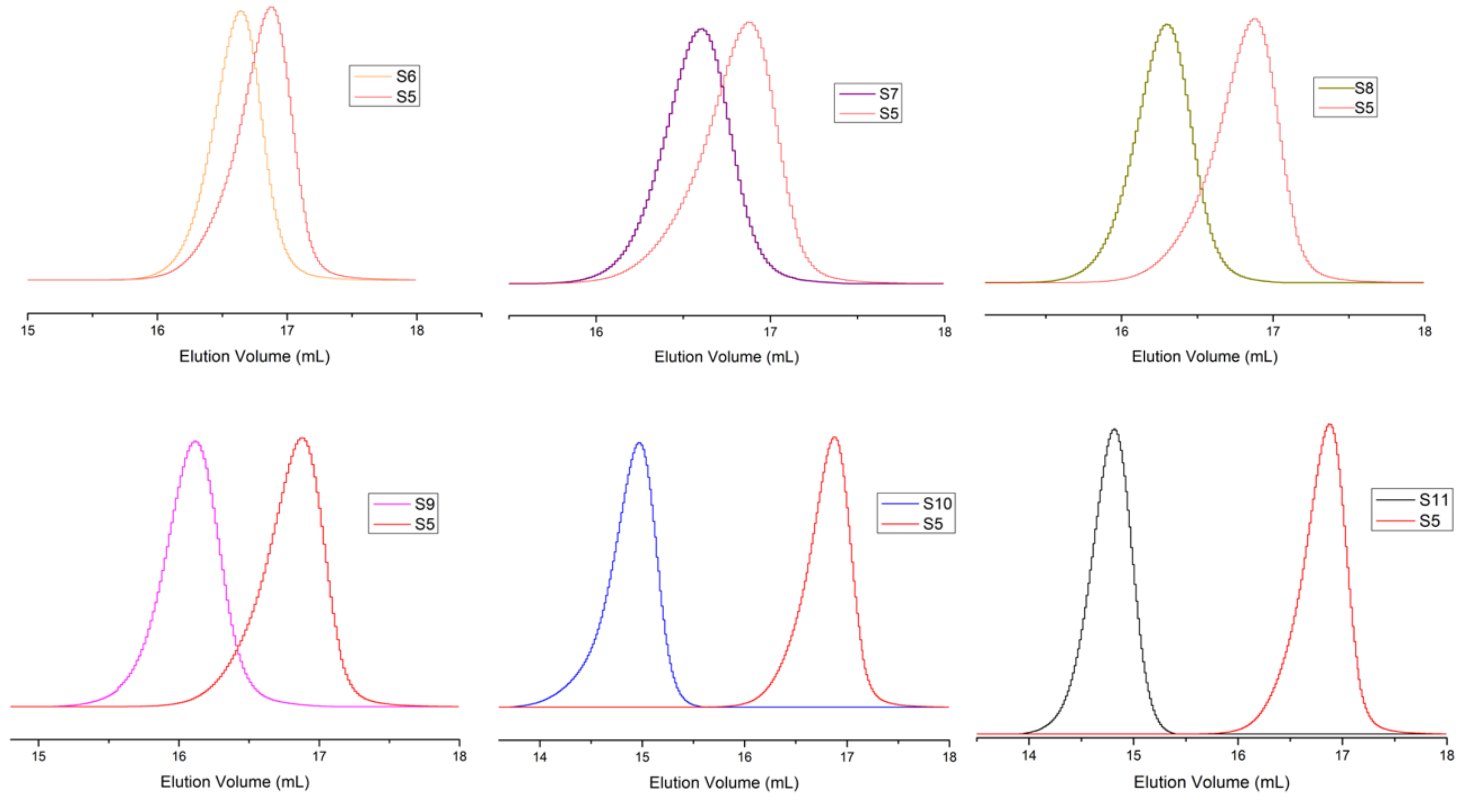

Figure S4. GPC traces of 8-arm star PBLG (S5) and polypept(o)ide star polymers S6-S11.

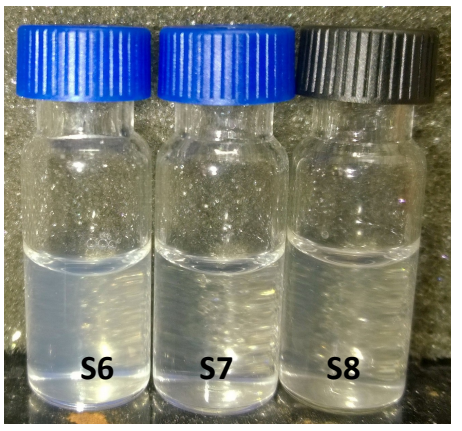

Figure S5. Solutions of star polypept(o)ides S6-S8 in water $(5 \mathrm{mg} / \mathrm{mL})$

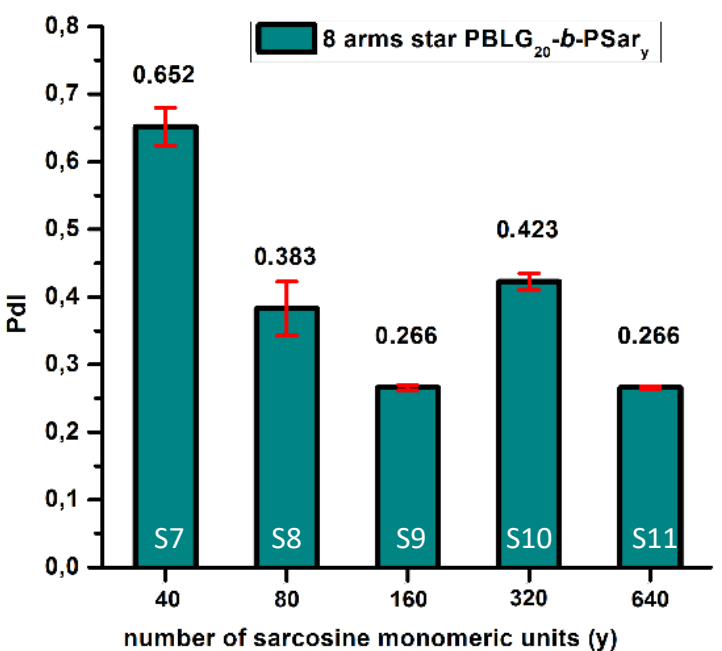

Figure S6. Dispersity index $(P d I)$ of star polypept(o)ides S7-S11 at a concentration of $5 \mathrm{mg} / \mathrm{mL}$ in water (error bar represents the standard deviation of three measurements). 
Table S1. Hydrodynamic radius $\left(R_{H}\right)$ and dispersity index (PdI) of star polymer nanoaggregates in deionized water (error values represent the standard deviation of three measurements).

\begin{tabular}{|c|c|c|}
\hline \multirow{2}{*}{ Sample } & \multicolumn{2}{|c|}{$10 \mathrm{mg} / 1 \mathrm{ml}$} \\
\cline { 2 - 3 } & RH (nm) & PdI \\
\hline 8 arm star PBLG20-b-PSAR20 & $230.7 \pm 11.2$ & $0.89 \pm 0.04$ \\
\hline 8 arm star PBLG20-b-PSAR 40 & $434.4 \pm 12.1$ & $0.61 \pm 0.04$ \\
\hline 8 arm star PBLG20-b-PSAR 80 & $311.1 \pm 4.7$ & $0.4 \pm 0.02$ \\
\hline 8 arm star PBLG20-b-PSAR 160 & $179.2 \pm 0.9$ & $0.32 \pm 0.04$ \\
\hline 8 arm star PBLG20-b-PSAR 320 & $131.9 \pm 1.9$ & $0.44 \pm 0.01$ \\
\hline 8 arm star PBLG20-b-PSAR640 & $124.9 \pm 4.2$ & $0.27 \pm 0.002$ \\
\hline
\end{tabular}

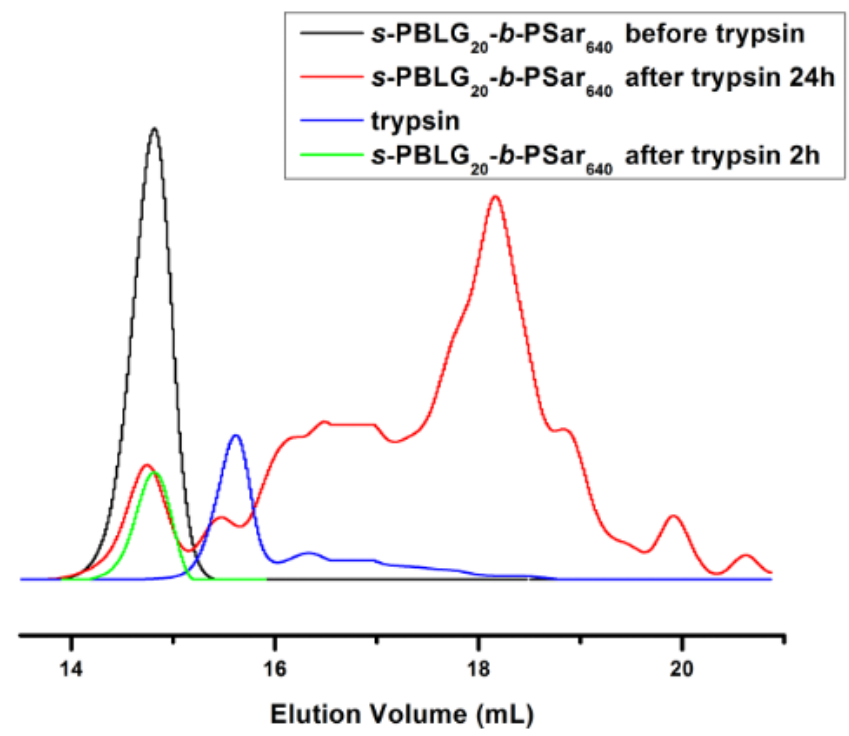

Figure S7. SEC traces of S11 before and after incubation with trypsin. 

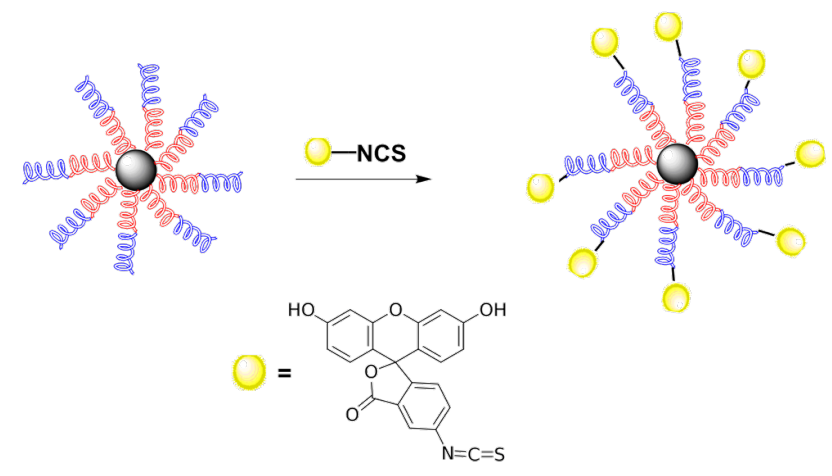

Figure S8. Labelling of S11 with fluorescein isothiocyanate, FITC.
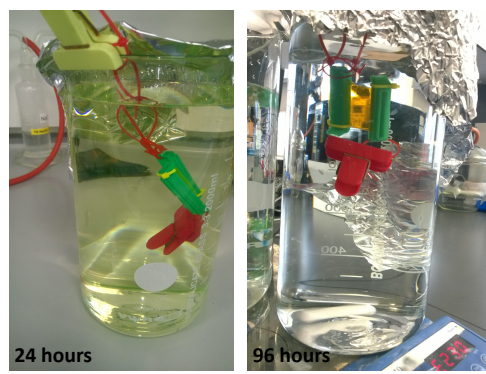

\begin{tabular}{|c|c|}
\hline Time (hours) & Absorbance (A) \\
\hline 24 & 0.145 \\
\hline 48 & 0.003 \\
\hline 72 & 0.005 \\
\hline 96 & 0.001 \\
\hline
\end{tabular}

Figure S9. Left: Images of dialysis to remove excess FITC from S11 after 24 and 96 hours. Right: $\mathrm{UV}-\mathrm{Vis}(\lambda=495 \mathrm{~nm})$ absorbance of dialysate demonstrating removal of excess FITC.

Table S2. Experimental data for the mass transport of FITC-S11 through artificial mucus (FITCS11 + Mucus) and controls without mucus (FITC-S11 - Mucus).

\begin{tabular}{|c|c|c|c|c|c|}
\hline & \multicolumn{4}{|c|}{ Cumulative transport $(\mu \mathrm{g})$} & \multirow[t]{2}{*}{$\begin{array}{c}\text { Percentage o } \\
\text { theoretical }\end{array}$} \\
\hline & $60 \mathrm{~min}$ & $120 \mathrm{~min}$ & $180 \mathrm{~min}$ & $240 \mathrm{~min}$ & \\
\hline \multicolumn{6}{|c|}{ Experiment 1} \\
\hline FITC-S11 + Mucus 1 & 0.38 & 1.88 & 3.20 & 3.53 & 35.25 \\
\hline FITC-S11 + Mucus 2 & 0.26 & 1.32 & 2.05 & 2.39 & 23.85 \\
\hline FITC-S11 - Mucus 1 & 2.76 & 6.27 & 7.59 & 8.31 & 83.12 \\
\hline FITC-S11 - Mucus 2 & 2.74 & 6.45 & 7.60 & 8.18 & 81.84 \\
\hline \multicolumn{6}{|c|}{ Experiment 2} \\
\hline FITC-S11 + Mucus 1 & 1.32 & 2.27 & 3.05 & 4.04 & 40.37 \\
\hline FITC-S11 + Mucus 2 & 2.23 & 3.41 & 4.25 & 5.38 & 53.81 \\
\hline FITC-S11 - Mucus 1 & 2.16 & 10.45 & 9.35 & 10.19 & 101.87 \\
\hline FITC-S11 - Mucus 2 & 3.08 & 5.65 & 6.82 & 8.18 & 81.78 \\
\hline \multicolumn{6}{|c|}{ Experiment 3} \\
\hline FITC-S11 + Mucus 1 & 4.30 & 5.49 & 6.77 & 7.55 & 75.52 \\
\hline FITC-S11 + Mucus 2 & 1.39 & 3.52 & 5.01 & 8.33 & 83.28 \\
\hline FITC-S11 - Mucus 1 & 6.24 & 8.25 & 9.41 & 9.78 & 97.84 \\
\hline FITC-S11 - Mucus 2 & 5.90 & 8.47 & 9.47 & 9.97 & 99.68 \\
\hline
\end{tabular}




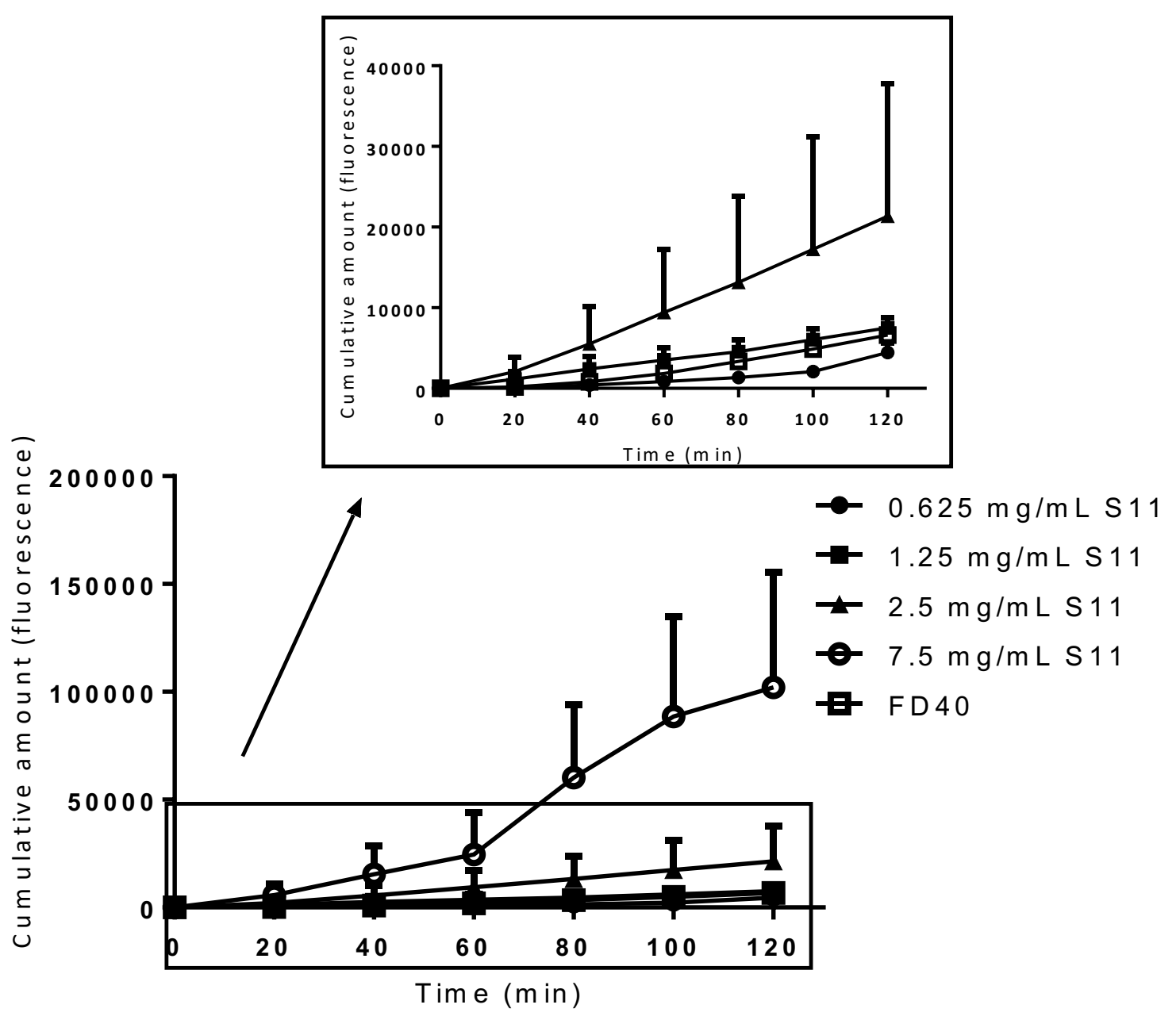

Figure S10. Cumulative permeated signal for 0.625-7.5 mg/mL FITC-S11 (S11) and FD40 (2.5 $\mathrm{mg} / \mathrm{mL})$ across rat jejunal mucosae mounted in Franz Cells $(\mathrm{n}=3-4)$. Inset shows magnification of groups in the absence of the $7.5 \mathrm{mg} / \mathrm{mL} \mathrm{S11}$ group. 